\title{
STACKED SUBMODULES OF TORSION MODULES OVER DISCRETE VALUATION DOMAINS
}

\author{
Pudji Astuti and Harald K. Wimmer
}

\begin{abstract}
A submodule $W$ of a torsion module $M$ over a discrete valuation domain is called stacked in $M$ if there exists a basis $\mathcal{B}$ of $M$ such that multiples of elements of $\mathcal{B}$ form a basis of $W$. We characterise those submodules which are stacked in a pure submodule of $M$.
\end{abstract}

\section{INTRODUCTION}

Let $R$ be a discrete valuation domain and let $p$ be a prime element of $R$ such that $R p$ is the maximal ideal of $R$. Let $M$ be a torsion module over $R$ and let $W$ be a submodule of $M$. In accordance with [7] and [6] we call a set $\left\{u_{\kappa} \mid \kappa \in K\right\}$ a basis of $M$ if $M=\bigoplus_{\kappa \in K} R u_{\kappa}$. We say that $W$ is stacked in $M$ if there exists a basis $\mathcal{X}=\left\{x_{\lambda} \mid \lambda \in \Lambda\right\}$ of $W$ and a basis $\mathcal{U}=\left\{u_{\kappa} \mid \kappa \in K\right\}$ of $M$ such that $\Lambda \subseteq K$ and $x_{\lambda}=p^{t_{\lambda}} u_{\lambda}$ for suitable nonnegative integers $t_{\lambda}$. In that case we call $\mathcal{X}$ a stacked basis of $W$ ([4]). If $M$ is of bounded order, that is, if there exists a positive integer $m$ such that $p^{m} x=0$ for all $x \in M$, then it is known [7, p. 65] that $W$ is stacked in $M$ if and only if

$$
p^{n} W \cap p^{n+r} M=p^{n}\left(W \cap p^{r} M\right)
$$

holds for all $n \geqslant 0, r \geqslant 0$. In general however, if $M$ is not of bounded order then condition (1.1) alone need not imply that $W$ is stacked in $M$ (see Exercise 78(b) in [7, p. 65]). In this paper we shall characterise those submodules which are stacked in a pure submodule of $M$.

Throughout this paper the letters $\mathcal{U}, \mathcal{V}, \mathcal{X}, \ldots$, will denote subsets of $M$. We shall use the letters $u, v, x, \ldots$, for elements of the module $M$, and $\alpha, \beta, \mu, \ldots$, will be elements of the ring $R$. Using the terminology for Abelian $p$-groups in [6, p. 4] we say that $x \in M$ has exponent $k$, and we write $\mathrm{e}(x)=k$, if $k$ is the smallest nonnegative integer such that $p^{k} x=0$. Clearly, $\mathrm{e}(0)=0$. An element $x \in M$ is said to have (finite) height $s$ if $x \in p^{s} M$

Received 28th April, 2003

The research of the first author was supported by Deutscher Akademischer Austauschdienst under Award No. A/98/25636. The authors would like to thank O. Mutzbauer and W. Schmale for valuable references.

Copyright Clearance Centre, Inc. Serial-fee code: 0004-9727/03 \$A2.00+0.00. 
and $x \notin p^{s+1} M$. In this case we write $\mathrm{h}(x)=s$. We set $\mathrm{h}(x)=\infty$ if $x \in p^{s} M$ for all $s \geqslant 0$. Thus $\mathrm{h}(0)=\infty$. Note that the height of all nonzero elements of $M$ is bounded if and only if $M$ is of bounded order.

Let $\langle\mathcal{X}\rangle$ be the submodule spanned by $\mathcal{X}$. When we write

$$
\alpha_{1} x_{1}+\cdots+\alpha_{m} x_{m} \in\langle\mathcal{X}\rangle
$$

we tacitely assume $x_{i} \in \mathcal{X}$ and $\alpha_{i} x_{i} \neq 0, i=1, \ldots, m$, and $x_{i} \neq x_{j}$ if $i \neq j$.

Let $R^{*}$ be the group of units of $R$. We set $\alpha \sim \beta$ if $\alpha=\beta \varepsilon$ for some $\varepsilon \in R^{*}$. It will be convenient to write $\mathrm{h}(\alpha)=s$ if $\alpha \sim p^{s}$. Let us recall the following properties of the height function on $M$ (see for example, [6, p. 154]). For all $x, y \in M$ we have $\mathbf{h}(p x) \geqslant \mathbf{h}(x)+1$, and

$$
\mathrm{h}(x+y) \geqslant \min \{\mathrm{h}(x), \mathrm{h}(y)\}
$$

Hence

$$
\mathrm{h}(\alpha x) \geqslant \mathrm{h}(\alpha)+\mathrm{h}(x) \text { for all } \alpha \in R .
$$

We say that an element $x$ is h-regular if $\mathrm{h}(x)=\infty$ or if $\mathrm{h}(x)$ is finite and

$$
\mathrm{h}(\alpha x)=\mathrm{h}(\alpha)+\mathrm{h}(x) \text { for all } \alpha \text { with } \mathrm{h}(\alpha)<\mathrm{e}(x) \text {. }
$$

Property (1.4) can be traced back to Baer [2]. In [2, p. 484] an element $x$ of an Abelian $p$-group is called regular if $\mathrm{h}(x)=\infty$ or if $\mathrm{h}(x)=k<\infty$ and

$$
\mathrm{e}(x)+\mathrm{h}(x)=\cdots=\mathrm{e}\left(p^{k-1} x\right)+h\left(p^{k-1} x\right) .
$$

As usual, a set $\mathcal{X}$ is called independent if $0 \notin \mathcal{X}$ and if for any finite subset $\left\{x_{1}, \ldots, x_{m}\right\}$ of $\mathcal{X}$ a relation $\alpha_{1} x_{1}+\cdots+\alpha_{m} x_{m}=0$ implies $\alpha_{i} x_{i}=0, i=1, \ldots, m$. We shall employ two stronger concepts of independence. The first one is adapted from Fuchs [5]. We call a set $\mathcal{X} p$-independent (or pure independent) if it is independent and contains no elements of infinite height, and if

$$
\alpha_{1} x_{1}+\cdots+\alpha_{m} x_{m} \in\langle\mathcal{X}\rangle
$$

implies

$$
\mathrm{h}\left(\alpha_{1} x_{1}+\cdots+\alpha_{m} x_{m}\right)=\min \left\{\mathrm{h}\left(\alpha_{i}\right) \mid i=1, \ldots, m\right\} .
$$

The other definition is motivated by the inequality

$$
\mathrm{h}\left(\alpha_{1} x_{1}+\cdots+\alpha_{m} x_{m}\right) \geqslant \min \left\{\mathrm{h}\left(\alpha_{i}\right)+\mathrm{h}\left(x_{i}\right) \mid i=1, \ldots, m\right\},
$$

which follows from (1.3) and (1.2). We say that $\mathcal{X}$ is $h$-independent if $\mathcal{X}$ is independent and (1.6) implies

$$
\mathrm{h}\left(\alpha_{1} x_{1}+\cdots+\alpha_{m} x_{m}\right)=\min \left\{\mathrm{h}\left(\alpha_{i}\right)+\mathrm{h}\left(x_{i}\right) \mid i=1, \ldots, m\right\} .
$$


Our concept of $h$-independence combines properties used in [3] to describe extendible Jordan bases of marked subspaces. It is obvious that a set $\mathcal{X}$ is $p$-independent if and only if it is $h$-independent and all of its elements have height zero.

For the elements $x$ of a submodule $S$ of $M$ we may define $\mathrm{h}_{S}(x)$ as the height of $x$ in $S$. We always have $\mathrm{h}_{S}(x) \leqslant \mathrm{h}(x)$. A submodule $S$ of $M$ is called pure in $M$ if $\mathrm{h}_{S}(x)=\mathrm{h}(x)$ for all $x \in S$, or equivalently if $S \cap p^{i} M=p^{i} S$ for all $i \geqslant 0$. The following lemma is due to Fuchs [5].

Lemma 1.1. For a set $\mathcal{X}$ the following conditions are equivalent.

(i) $\mathcal{X}$ is p-independent.

(ii) $\mathcal{X}$ is independent and the submodule $\langle\mathcal{X}\rangle$ is pure in $M$.

Since $M$ is pure in itself it follows from the preceding lemma that a basis of $M$ is $p$-independent. It is also obvious that all nonzero elements of $M$ have finite height if $M$ has a basis.

Our main result is the following theorem. It will be proved in Section 3 together with a corollary.

THEOREM 1.2. Let $M$ be a torsion module over a discrete valuation domain and let $W$ be a submodule of $M$. The following statements are equivalent.

(i) There exists a pure submodule $S$ of $M$ such that $W$ is stacked in $S$.

(ii) $W$ has an $h$-independent basis.

It will be shown in Proposition 3.3 that condition (1.1) is necessary for the existence of an $h$-independent basis of $W$. In the case where $M$ is of bounded order we note the following result.

Corollary 1.3. Let $M$ of bounded order. For a submodule $W$ of $M$ the following statements are equivalent.

(i) $W$ is stacked in $M$.

(ii) $W$ has an $h$-independent basis.

(iii) Condition (1.1) holds.

It is well-known [1] that the Jordan normal form can be studied in the framework of the theory of finitely generated modules over a principal ideal domain. Hence [7, Exercise 79, p. 65], and Theorem 1.2 and its proof provide an alternative access to results in [3] on extensions of Jordan bases for invariant subspaces of a matrix.

\section{2. $h$-INDEPENDENCE}

This section contains the results on $h$-independence which we shall need in the course of the proof of Theorem 1.2. We shall make constant use of the following observations 
on the height function. Suppose $p^{m} x \neq 0$ and $\mathrm{h}\left(p^{m} x\right)=m+r$. Then we have $\mathrm{h}(x) \leqslant r$. If $x \neq 0$ is an element with $\mathrm{h}(x)=s$ and $\mathrm{e}(x)=k$ then $x$ is $h$-regular if and only if

$$
\mathrm{h}\left(p^{j} x\right)=j+\mathrm{h}(x), j=1, \ldots, k-1,
$$

or equivalently, if and only if

$$
\mathrm{h}\left(p^{k-1} x\right)=(k-1)+\mathrm{h}(x)
$$

or equivalently, $p^{j} x$ is $h$-regular for all $j \geqslant 0$.

It is not difficult to see that an independent set $\mathcal{X}$ is $h$-independent if and only if its elements are $h$-regular, and if $x=\alpha_{1} x_{1}+\cdots+\alpha_{m} x_{m} \in\langle\mathcal{X}\rangle, x \neq 0$, then $\mathrm{h}(x)$ $=\min \left\{\mathrm{h}\left(\alpha_{i} x_{i}\right) ; i=1, \ldots, m\right\}$ for all $\alpha_{i} \in R$.

It is obvious that $h(x) \neq h(y)$ implies $h(x+y)=\min \{h(x), h(y)\}$. Hence if a strict inequality $\mathrm{h}(x+y)>\min \{\mathrm{h}(x), \mathrm{h}(y)\}$ holds, then $\mathrm{h}(x)=\mathrm{h}(y)$. Therefore, whenever we want to show that an independent set $\left\{x_{1}, \ldots, x_{m}\right\}$ of $h$-regular elements is $h$-independent we have to make sure that $\mathrm{h}\left(\alpha_{i} x_{i}\right)=r, i=1, \ldots, m$, implies $\mathrm{h}\left(\alpha_{1} x_{1}+\cdots+\alpha_{m} x_{m}\right) \leqslant r$.

We shall also make frequent use of the following fact.

LEMMA 2.1. Let $\mathcal{X}=\mathcal{X}_{1} \cup \cdots \cup \mathcal{X}_{k}$ be a disjoint union of $h$-independent sets. Then $\mathcal{X}$ is $h$-independent if and only if $x_{i_{\tau}} \in\left\langle\mathcal{X}_{i_{\tau}}\right\rangle, x_{i_{\tau}} \neq 0$, and $1 \leqslant i_{1}<\cdots<i_{t} \leqslant m$ imply that $\left\{x_{i_{1}}, \ldots, x_{i_{t}}\right\}$ is $h$-independent.

In the following observation we are concerned with a submodule where all elements are $h$-regular.

LEMma 2.2. Let $\mathcal{X}$ be $h$-independent and assume that

$$
\mathrm{h}(x)+\mathrm{e}(x)=t \text { for all } x \in \mathcal{X} \text {. }
$$

Then each nonzero element $y \in\langle\mathcal{X}\rangle$ is $h$-regular and

$$
\mathrm{h}(y)+\mathrm{e}(y)=t .
$$

Proof: If $x \in \mathcal{X}$ then $x$ is $h$-regular, and we have

$$
\mathrm{h}(\alpha x)+\mathrm{e}(\alpha x)=\mathrm{h}(x)+\mathrm{e}(x)=t
$$

if $\mathrm{h}(\alpha)<\mathrm{e}(x)$. Let $y=\alpha_{1} x_{1}+\cdots+\alpha_{m} x_{m} \in\langle\mathcal{X}\rangle$ be nonzero with $\mathrm{h}(y)=r$ and $\mathrm{e}(y)=k$. Assume $\mathrm{h}\left(\alpha_{1} x_{1}\right)=\min \left\{\mathrm{h}\left(\alpha_{i} x_{i}\right) \mid i=1, \ldots, m\right\}$. Since $\mathcal{X}$ is $h$-independent we have $\mathrm{h}\left(\alpha_{1} x_{1}\right)=r$. Then (2.9) implies $\mathrm{e}\left(\alpha_{1} x_{1}\right)=t-r$. From $r \leqslant \mathrm{~h}\left(\alpha_{i} x_{i}\right)$ we obtain $\mathrm{e}\left(\alpha_{i} x_{i}\right) \leqslant t-r$. Hence $\mathrm{e}(y) \leqslant t-r$. Since $\mathcal{X}$ is independent it follows from $p^{k} y=0$ that $p^{k} \alpha_{i} x_{i}=0$ for all $i$. For $i=1$ we obtain $k \geqslant \mathrm{e}\left(\alpha_{1} x_{1}\right)=t-r$, and we deduce $k=\mathrm{e}(y)=t-\mathrm{h}(y)$. Since $y$ was an arbitrary element of $\langle\mathcal{X}\rangle$ it follows that $\mathrm{h}(\alpha y)+\mathrm{e}(\alpha y)=t$ for all $\alpha \neq 0$. Therefore $y$ is $h$-regular.

The subsequent criterion for $h$-independence may be of interest in its own right. 
LEMмA 2.3. If $\mathcal{Y}=\left\{y_{0}, y_{1}, \ldots, y_{m}\right\} \subseteq M$ is a set of $h$-regular elements such that

$$
\mathrm{h}\left(y_{0}\right)+\mathrm{e}\left(y_{0}\right)>\cdots>\mathrm{h}\left(y_{m}\right)+\mathrm{e}\left(y_{m}\right)
$$

then $\mathcal{Y}$ is $h$-independent.

Proof: We proceed by induction on $|\mathcal{Y}|$. Set $\mathrm{h}\left(y_{i}\right)=s_{i}$ and $\mathrm{e}\left(y_{i}\right)=k_{i}$, $i=0,1, \ldots, m$. Assume that $\tilde{\mathcal{Y}}=\left\{y_{1}, \ldots, y_{m}\right\}$ is $h$-independent. Let $y_{0}$ be $h$-regular satisfying

$$
s_{0}+k_{0}>s_{j}+k_{j}, j=1, \ldots, m \text {. }
$$

Let us show first that $\mathcal{Y}=\left\{y_{0}\right\} \cup \tilde{\mathcal{Y}}$ is an independent set. Suppose the contrary such that there exists a nonzero element of the form

$$
\alpha_{0} y_{0}=\alpha_{1} y_{1} \cdots+\alpha_{m} y_{m}
$$

Since $\tilde{\mathcal{Y}}$ is independent we have $\alpha_{j} y_{j} \neq 0, j \geqslant 1$, and

$$
\mathrm{e}\left(\alpha_{0} y_{0}\right)=\max _{j \geqslant 1}\left\{\mathrm{e}\left(\alpha_{j} y_{j}\right)\right\}
$$

Set $\mathrm{h}\left(\alpha_{0} y_{0}\right)=r$. Then $\mathrm{h}\left(\alpha_{0} y_{0}\right)+\mathrm{e}\left(\alpha_{0} y_{0}\right)=s_{0}+k_{0}$ yields $\mathrm{e}\left(\alpha_{0} y_{0}\right)=s_{0}+k_{0}-r$, and (2.10) implies

$$
\mathrm{e}\left(\alpha_{0} y_{0}\right)>\mathrm{e}\left(\alpha_{j} y_{j}\right)+\mathrm{h}\left(\alpha_{j} y_{j}\right)-r \geqslant \mathrm{e}\left(\alpha_{j} y_{j}\right)+\left[\min _{j \geqslant 1}\left\{\mathrm{~h}\left(\alpha_{j} y_{j}\right)\right\}-r\right], j \geqslant 1 .
$$

Since $\widetilde{\mathcal{Y}}$ is $h$-independent it follows from (2.12) that

$$
r=\mathrm{h}\left(\alpha_{0} y_{0}\right)=\min _{j \geqslant 1}\left\{\mathrm{~h}\left(\alpha_{j} y_{j}\right)\right\}
$$

Hence we obtain $\mathrm{e}\left(\alpha_{0} y_{0}\right)>\max \left\{\mathrm{e}\left(\alpha_{j} y_{j}\right) \mid j \geqslant 1\right\}$, in contradiction to (2.13). Now let us turn to $h$-independence of $\mathcal{Y}$. Let $y=\alpha_{0} y_{0}+\alpha_{1} y_{1} \cdots+\alpha_{m} y_{m}$ be nonzero, and $\mathrm{h}\left(\alpha_{i} y_{i}\right)=r, i \geqslant 0$. Then $\mathrm{e}\left(\alpha_{i} y_{i}\right)=k_{i}+s_{i}-r, i \geqslant 0$, and by $(2.10)$ we obtain $\mathrm{e}\left(\alpha_{j} y_{j}\right)$ $<k_{0}+s_{0}-r, j \geqslant 1$. Hence

$$
p^{k_{0}+s_{0}-r-1} y=p^{k_{0}+s_{0}-r-1} \alpha_{0} y_{0} \neq 0
$$

Since $y_{0}$ is $h$-regular and $\mathrm{h}\left(\alpha_{0} y_{0}\right)=r$ it is clear that

$$
\mathrm{h}\left(p^{k_{0}+s_{0}-r-1} \alpha_{0} y_{0}\right)=k_{0}+s_{0}-1,
$$

and therefore $\mathrm{h}(y) \leqslant r$. 


\section{Partitions of bases}

For the proof of Theorem 1.2 it will be crucial that $h$-independence of a set $\mathcal{X}$ can be checked by examining suitably chosen classes of subsets.

LEMMA 3.1 .

(i) $A$ set $\mathcal{X}$ is $h$-independent if the sets

$$
\mathcal{X}^{[t]}=\{x \in \mathcal{X} ; \mathrm{e}(x)+\mathrm{h}(x)=t\},
$$

$t \geqslant 1$, are $h$-independent.

(ii) Let $\mathcal{U}$ be a set of elements of height zero. Then $\mathcal{U}$ is p-independent if the sets

$$
\mathcal{U}_{k}=\{u \in \mathcal{U} ; \mathrm{e}(u)=k\},
$$

$k \geqslant 1$, are $p$-independent.

(iii) Let $\mathcal{Z}$ be a set of elements of exponent 1. Then $\mathcal{Z}$ is $h$-independent if the sets

$$
\mathcal{Z}^{s-1}=\{z \in \mathcal{Z} ; \mathrm{h}(z)=s-1\},
$$

$s \geqslant 1$, are $h$-independent.

Proof: (i) It suffices to show that for a given $k$ the set $\cup\left\{\mathcal{X}^{[i]} ; 1 \leqslant i \leqslant k\right\}$ is $h$-independent. Let $\widetilde{\mathcal{X}}=\left\{x_{i_{1}}, \ldots, x_{i_{t}}\right\}$ be such that $x_{i_{\tau}} \in\left\langle\mathcal{X}^{\left[i_{\tau}\right]}\right\rangle, x_{i_{\tau}} \neq 0$, and 1 $\leqslant i_{1}<\cdots<i_{t} \leqslant m$. We know from Lemma 2.2 that $x_{i_{\tau}}$ is $h$-regular and $\mathrm{h}\left(x_{i_{\tau}}\right)$ $+\mathrm{e}\left(x_{i_{\tau}}\right)=i_{r}$. Hence Lemma 2.3 implies that $\widetilde{\mathcal{X}}$ is $h$-independent and Lemma $2.1 \mathrm{ex}-$ tends $h$-independence from $\tilde{\mathcal{X}}$ to $\mathcal{X}$.

For (ii) and (iii) we note that $\mathcal{U}^{[k]}=\mathcal{U}_{k}$ and $\mathcal{Z}^{[s]}=\mathcal{Z}^{s-1}$.

Using the preceding lemma we can relate a set $\mathcal{X}$ and its $h$-independence to a corresponding set $\mathcal{U}$ of height zero elements and to a subset $\mathcal{Z}$ of the socle of $M$.

Proposition 3.2. Let $\mathcal{X}=\left\{x_{\lambda} \mid \lambda \in \Lambda\right\}$ be a an independent subset of $M$ such that $\mathrm{h}\left(x_{\lambda}\right)=s_{\lambda}, \mathrm{e}\left(x_{\lambda}\right)=k_{\lambda}, \lambda \in \Lambda$. Let $\mathcal{U}=\left\{u_{\lambda} \mid \lambda \in \Lambda\right\}$ be a corresponding set of height zero elements of $M$ such that $x_{\lambda}=p^{s_{\lambda}} u_{\lambda}, \lambda \in \Lambda$. Then the following statements are equivalent.

(i) $\mathcal{X}$ is $h$-independent.

(ii) $\mathcal{U}$ is $p$-independent.

(iii) The set $\mathcal{Z}=\left\{z_{\lambda}=p^{k_{\lambda}-1} x_{\lambda} \mid \lambda \in \Lambda\right\}$ is h-independent.

Proof: Since $\mathcal{X}$ is independent we have $x_{\lambda} \neq x_{\mu}$ and $u_{\lambda} \neq u_{\mu}$, if $\lambda \neq \mu$. For $\lambda \in \Lambda$ define $\pi x_{\lambda}=u_{\lambda}$. Then $\pi: \mathcal{X} \rightarrow \mathcal{U}$ is a bijection. Note that $x_{\lambda}$ is $h$-regular if and only if $u_{\lambda}=\pi x_{\lambda}$ is $h$-regular. 
(i) $\Rightarrow$ (ii) Because of Lemma 3.1 it suffices to prove that the sets $\mathcal{U}_{k}$ in (3.14) are $p$-independent. Consider an element

$$
v=\alpha_{1} u_{1}+\cdots \alpha_{m} u_{m} \in\left\langle\mathcal{U}_{k}\right\rangle
$$

with

$$
r=\mathrm{h}\left(\alpha_{1}\right)=\cdots=\mathrm{h}\left(\alpha_{t}\right)<\mathrm{h}\left(\alpha_{t+1}\right) \leqslant \cdots \leqslant \mathrm{h}\left(\alpha_{m}\right)<k
$$

Then

$$
\alpha_{j}=p^{r} \gamma_{j}, \gamma_{j} \sim 1, \quad \text { for } j=1, \ldots, t
$$

Let $x_{j} \in \mathcal{X}$ be such that $\pi x_{j}=u_{j}$ and $x_{j}=p^{\mu_{j}} u_{j}, j=1, \ldots, t$. Then $\mu_{j}<k=\mathrm{e}\left(u_{j}\right)$. Hence $k-\mu_{j}-1 \geqslant 0$ and

$$
p^{k-1} \gamma_{j} u_{j}=p^{k-1-\mu_{j}} \gamma_{j} p^{\mu_{j}} u_{j}=p^{k-1-\mu_{j}} \gamma_{j} x_{j} \neq 0, j=1, \ldots, t .
$$

Because of (3.15) and (3.16) we have

$$
\begin{aligned}
p^{k-r-1} v=p^{k-r-1}\left(\alpha_{1} u_{1}+\cdots+\alpha_{t} u_{t}\right)=p^{k-1}\left(\gamma_{1} u_{1}\right. & \left.+\cdots+\gamma_{t} u_{t}\right) \\
& =p^{k-1-\mu_{1}} \gamma_{1} x_{1}+\cdots+p^{k-1-\mu_{t}} \gamma_{t} x_{t}
\end{aligned}
$$

Recall that $\tilde{\mathcal{X}}=\left\{x_{1}, \ldots, x_{t}\right\} \subseteq \mathcal{X}$ is $h$-independent. Hence it follows from (3.17) that $p^{k-1-r} v \neq 0$. In particular we have $v \neq 0$. Thus $\mathcal{U}_{k}$ is independent. We also obtain

$$
\begin{aligned}
\mathrm{h}\left(p^{k-1-r} v\right) & =\min \left\{\mathrm{h}\left(p^{k-1-\mu_{j}} \gamma_{j} x_{j}\right) \mid 1 \leqslant j \leqslant t\right\} \\
& =\min \left\{\mathrm{h}\left(p^{k-1} \gamma_{j} u_{j}\right) \mid 1 \leqslant j \leqslant t\right\}=k-1 .
\end{aligned}
$$

Hence $\mathrm{h}(v) \leqslant r$, which implies

$$
\mathrm{h}(v)=r=\min \left\{\mathrm{h}\left(\alpha_{i}\right) \mid 1 \leqslant i \leqslant m\right\} .
$$

Thus $\mathcal{U}_{k}$ is $h$-independent.

(ii) $\Rightarrow$ (i) Assume that $\mathcal{U}$ is $p$-independent. Let us focus on an element $x=\alpha_{1} x_{1}$ $+\cdots+\alpha_{m} x_{m} \in\langle\mathcal{X}\rangle$ with $\mathrm{h}\left(x_{i}\right)=s_{i}$ and $u_{i}=\pi x_{i}, 1 \leqslant i \leqslant m$. From $x_{i}=p^{s_{i}} u_{i}$ and $\mathrm{h}\left(\alpha_{i} x_{i}\right)=\mathrm{h}\left(\alpha_{i} p^{s_{i}}\right)$ we obtain

$$
\mathrm{h}(x)=\mathrm{h}\left(\sum \alpha_{i} p^{s_{i}} u_{i}\right)=\min \left\{\mathrm{h}\left(\alpha_{i} p^{s_{i}}\right)\right\}=\min \left\{\mathrm{h}\left(\alpha_{i}\right)+\mathrm{h}\left(x_{i}\right)\right\}
$$

which shows that $\mathcal{X}$ is $h$-independent.

(ii) $\Leftrightarrow$ (iii) For $z_{\lambda}=p^{k_{\lambda}-1} x_{\lambda}$ set $\tilde{\pi} z_{\lambda}=u_{\lambda}$. Then $\tilde{\pi}: \mathcal{Z} \rightarrow \mathcal{U}$ is a bijection and we can apply the first part of the proposition to the case where $\mathcal{X}=\mathcal{Z}$.

We are now ready to derive our main result as an immediate consequence of Proposition 3.2 . 
ProOF OF THEOREM 1.2:

(i) $\Rightarrow$ (ii) Let $S$ be a pure submodule of $M$ with a basis $\mathcal{U}=\left\{u_{\lambda} \mid \lambda \in \Lambda\right\}$ such that $W$ has a basis $\mathcal{X}=\left\{p^{s_{\lambda}} u_{\lambda} \mid \lambda \in \Lambda\right\}$. We know from Lemma 1.1 that the set $\mathcal{U}$ is $p$-independent. Hence it follows from Proposition 3.2 that $\mathcal{X}$ is an $h$-independent basis of $W$.

(ii) $\Rightarrow$ (i) Let $\mathcal{X}=\left\{x_{\lambda} \mid \lambda \in \Lambda\right\}$ be an $h$-independent basis of $W$ and let $\mathcal{U}$ $=\left\{u_{\lambda} \mid \lambda \in \Lambda\right\}$ be a set of $h$-regular elements of height zero such that $x_{\lambda}=p^{s_{\lambda}} u_{\lambda}$. Then it follows from Proposition 3.2 that $\mathcal{U}$ is $p$-independent. Hence, by Lemma 1.1 the submodule $S=\langle\mathcal{U}\rangle$ is pure and $W$ is stacked in $S$.

Before turning to the proof of Corollary 1.3 we want to show that Kaplanski's condition (1.1) is necessary for the existence of an $h$-independent basis of $M$.

Proposition 3.3. If $W$ has an $h$-independent basis then $W$ satisfies (1.1).

Proof: It is obvious that (1.1) is equivalent to

$$
p^{n} W \cap p^{n+r} M \subseteq p^{n}\left(W \cap p^{r} M\right), n \geqslant 0, r \geqslant 0 .
$$

Take an element $x \in p^{n} W \cap p^{n+r} M$. We can assume that $r$ is maximal. Then $x=p^{n} w$ for some $w \in W$, and $\mathrm{h}(x)=n+r$. Now let $\mathcal{X}$ be an $h$-independent basis of $W$. Then $w=\alpha_{1} x_{1}+\cdots+\alpha_{m} x_{m} \in\langle\mathcal{X}\rangle$. Assume $\mathrm{e}\left(\alpha_{i} x_{i}\right)>n, i=1, \ldots, t$, and $\mathrm{e}\left(\alpha_{i} x_{i}\right) \leqslant n, i>t$. Set $\widetilde{w}=\alpha_{1} x_{1}+\cdots+\alpha_{t} x_{t}$. Then $\widetilde{w} \in W$ and $x=p^{n} \widetilde{w}$, and we obtain

$$
\begin{aligned}
n+r=\mathrm{h}\left(p^{n} w\right)=\min \left\{\mathrm{h}\left(p^{n} \alpha_{i} x_{i}\right) ; i=\right. & 1, \ldots, t\} \\
& =n+\min \left\{\mathrm{h}\left(\alpha_{i} x_{i}\right) ; i=1, \ldots, t\right\}=n+\mathrm{h}(\widetilde{w}) .
\end{aligned}
$$

Hence $\mathrm{h}(\widetilde{w})=r$. We have $\widetilde{w} \in p^{r} M$, and we conclude that $x \in p^{n}\left(W \cap p^{r} M\right)$.

Proof of Corollary 1.3: If $M$ is of bounded order then $M$ is a direct sum of cyclic submodules (see for example, $[7$, p. 88]) and each pure submodule is a direct summand of $M$. Hence the equivalence of (i) and (ii) follows immediately from Theorem 1.2. We refer to $[7$, p. 65]) for the fact that (i) and (iii) are equivalent provided that $M$ is of bounded order.

\section{REFERENCES}

[1] W.A. Adkins and St.H. Weintraub, Algebra: An approach via module theory, Graduate Texts in Mathematics 136 (Springer-Verlag, New York, 1992).

[2] R. Baer, 'Types of elements and the characteristic subgroups of abelian groups', Proc. London Math. Soc. 39 (1935), 481-514.

[3] R. Bru, L. Rodman and H. Schneider, 'Extensions of Jordan bases for invariant subspaces of a matrix', Linear Algebra Appl. 150 (1991), 209-225.

[4] J.M. Cohen and H. Gluck, 'Stacked bases for modules over principal ideal domains', $J$. Algebra 14 (1970), 493-505. 
[5] L. Fuchs, 'Notes on Abelian groups, II', Acta Math. Hungar. 11 (1960), 117-125.

[6] L. Fuchs, Infinite Abelian groups, Vol. I, Pure and Applied Mathematics 36 (Academic Press, New York, 1973).

[7] I. Kaplanski, Infinite Abelian groups (University of Michigan Press, Ann Arbor, MI, 1954).

Departemen Matematika Institut Teknologi Bandung

Bandung 40132

Indonesia

e-mail: pudji@dns.math.itb.ac.id
Mathematisches Institut

Universität Würzburg

D-97074 Würzburg

Germany

e-mail: wimmer@mathematik.uni-wuerzburg.de 